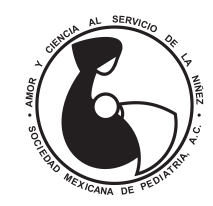

Vol. 87, No. 2 - Marzo-Abril 2020. pp 65-69 https://dx.doi.org/10.35366/94170

Revista Mexicana de

\title{
Neumonía adquirida en la comunidad y encefalitis por metapneumovirus humano
}

\author{
Ricardo Hernández-Sarmiento, ${ }^{\ddagger} *$ Martha I Álvarez-Olmos, ${ }^{\S}$ Cecilia Aguirre-Zambrano, ${ }^{\ddagger}$ \\ Daniel De Zubiria, ${ }^{\ddagger}$ María I Lozano-Jaramillo" \\ ‡ Universidad del Rosario; ${ }^{\S}$ Fundación Cardioinfantil, Universidades El Bosque, El Rosario, La Sabana; "| Fundación Cardioinfantil. \\ Bogotá DC, Colombia.
}

\begin{abstract}
RESUMEN
El metapneumovirus humano es un virus ARN, clasificado en el grupo de patógenos respiratorios como el segundo más frecuente de la lista, después del virus sincitial respiratorio. Por lo general, afecta a niños menores de cinco años y produce manifestaciones variables en las vías respiratorias superiores e inferiores. Asimismo, se ha asociado con el desarrollo de complicaciones neurológicas, lo que incrementa la morbilidad y mortalidad. Presentamos el caso de dos pacientes con sintomatología respiratoria asociada a alteraciones del sistema nervioso central dados por hiperreflexia, irritabilidad y convulsiones, con aislamiento de metapneumovirus humano por FilmArray ${ }^{\mathrm{TM}}$.
\end{abstract}

Palabras clave: Metapneumovirus, neumonía, encefalitis, niño.

\section{INTRODUCCIÓN}

La infección respiratoria aguda (IRA) es una de las mayores causas de morbimortalidad a nivel mundial. Puede afectar a niños sin discriminar el estrato socioeconómico, sin embargo, tiene una mayor mortalidad en países en vías de desarrollo. ${ }^{1}$

El metapneumovirus humano (hMPV, por sus siglas en inglés) es uno de los agentes etiológicos de la IRA que

\begin{abstract}
Human metapneumovirus is a RNA virus, classified in the group of respiratory pathogens, as the second most frequent after respiratory syncytial virus. It usually affects children under 5 years old and produces variable manifestations in the upper and lower respiratory tracts. Likewise, it has been associated with the development of neurological complications, which increses the morbidity and mortality rates. We present the case of two patients with respiratory symptoms associated with alterations of the central nervous system due to hyperreflexia, irritability and seizures. In these two cases human metapneumovirus was isolated by FilmArray $^{\mathrm{TM}}$.
\end{abstract}

Keywords: Metapneumovirus, pneumonia, encephalitis, child.

ha despertado atención en los últimos años, siendo la segunda causa más común de bronquiolitis y neumonía en niños menores de cinco años. ${ }^{2}$ Fue descubierto en 2001 en un paciente pediátrico $\mathrm{y}$, desde entonces, se aísla hasta en $16 \%$ de los pacientes con IRA. ${ }^{1,3}$

En virtud que hay pocos casos reportados similares, ${ }^{4,5}$ en este articulo describimos dos pacientes pediátricos en quienes se identificó infección por hMPV y tuvieron tanto IRA como trastornos neurológicos.

\footnotetext{
*Correspondencia: RHS, rhernandezs15@hotmail.com

Conflicto de intereses: Los autores declaran que no tienen.

Citar como: Hernández-Sarmiento R, Álvarez-Olmos MI, Aguirre-Zambrano C, De Zubiria D, Lozano-Jaramillo MI. Neumonía adquirida en la comunidad y encefalitis por metapneumovirus humano. Rev Mex Pediatr. 2020; 87(2):65-69. doi: 10.35366/94170

[Community-acquired pneumonia and encephalitis due to human metapneumovirus]
} 


\section{Caso clínico 1}

Paciente femenino de siete meses de edad llevada al servicio de Urgencias por cuadro clínico de ocho días de tos húmeda, emetizante, ocasionalmente cianosante, a lo cual seis días después se agregó fiebre de hasta $40{ }^{\circ} \mathrm{C}$, rinorrea hialina y secreción seropurulenta conjuntival.

$\mathrm{Al}$ examen físico se encontró febril, taquicárdica, hipoxémica, con signos de dificultad respiratoria moderada por tirajes intercostales, secreción conjuntival purulenta bilateral.

Se tomaron estudios de laboratorio en los cuales se observó la biometría sin leucocitosis, anemia normocítica e hipocrómica; mientras que la proteína $\mathrm{C}$ reactiva fue negativa. En la radiografía $(\mathrm{Rx})$ de tórax habían datos compatibles con neumonía, probablemente de etiología viral. Por lo anterior, se solicitó panel viral por inmunofluorescencia, el cual resultó negativo para virus sincitial respiratorio, influenza A y B y parainfluenza 1, 2 y 3.

La evolución clínica no fue satisfactoria, dado que persistía la dificultad respiratoria. Se sospechó coinfección bacteriana, por lo que se inició ampicilina/ sulbactam. Posteriormente presenta episodio de irritabilidad e hiperreflexia, por lo que ante la posibilidad de neuroinfección se realiza tomografía (TAC) de cráneo donde se evidencia realce meníngeo paraoccipital. El líquido cefalorraquídeo (LCR) no fue sugestivo de infección bacteriana, pero no se pudo descartar infección viral. La paciente se traslada a Unidad de Cuidados Intensivos Pediátricos (UCIP), donde se toma nueva Rx de tórax (Figura 1), así como TAC de tórax, la cual

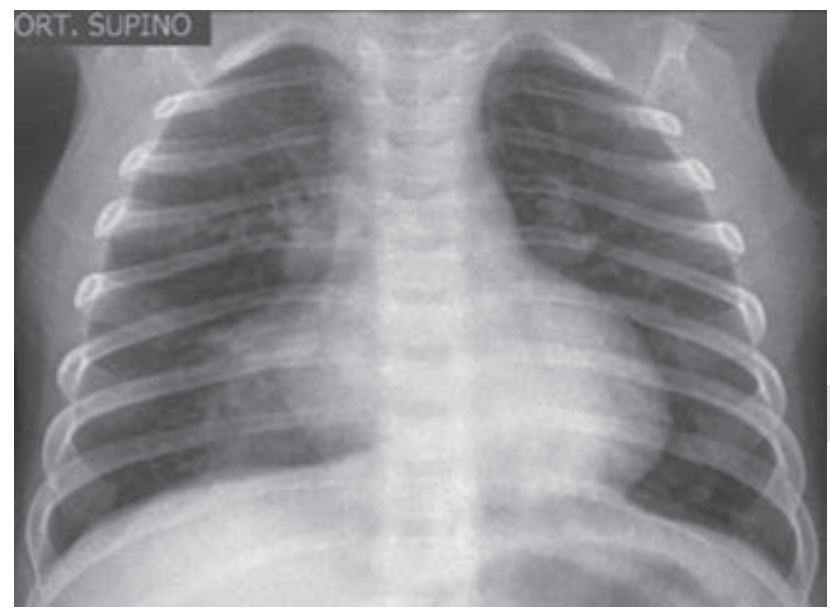

Figura 1: Rx tórax. Opacidades con tendencia a consolidar en lóbulo inferior derecho.
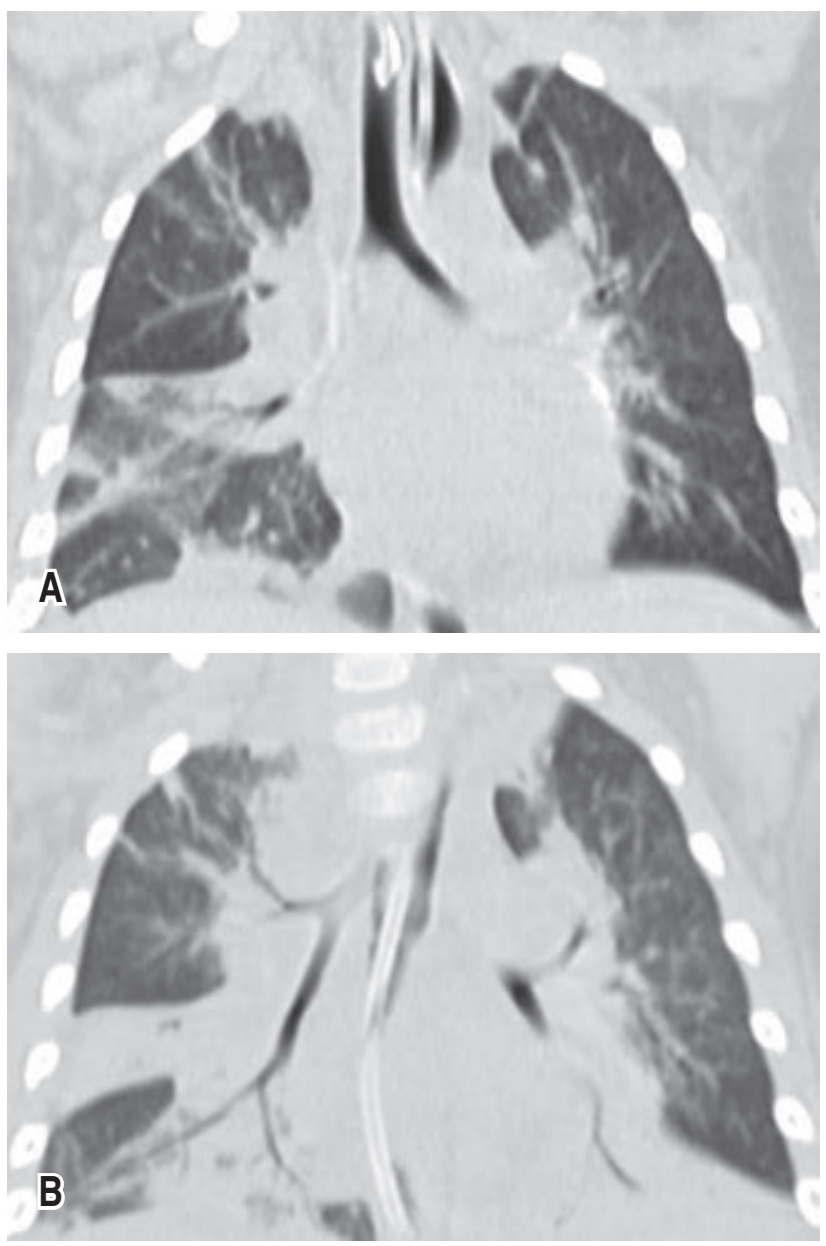

Figura 2: TAC tórax. Consolidaciones parenquimatosas con broncograma aéreo y vidrio esmerilado de ambos lóbulos inferiores, lóbulo superior izquierdo, región paratraqueal derecha del lóbulo superior derecho y lóbulo medio.

muestra datos de neumonía en diferentes lóbulos $(F i$ guras $2 A$ y B). Mediante FilmArray ${ }^{\mathrm{TM}}$ respiratorio se detectó hMPV.

Lentamente, la paciente fue mejorando manteniéndose con ventilación mecánica invasiva por siete días, egresándose después de dos meses de estancia hospitalaria, sin secuelas neurológicas.

\section{Caso clínico 2}

Paciente masculino de tres años previamente sano, que ingresa por cuadro de 24 horas de fiebre en $39^{\circ} \mathrm{C}$ asociada a rinorrea hialina y tos. $\mathrm{Al}$ examen físico de ingreso se detectó disminución de los ruidos respiratorios en hemitórax derecho. Presenta convulsión tónico-clónica 
de 15 minutos de duración. Requirió intubación orotraqueal por 14 días, así como estancia en UCIP por 19 días.

En la Rx tórax se observó ascenso de hemidiafragma derecho; mientras que la TAC de cráneo fue normal, lo mismo que hemograma. El LCR con hiperproteinorraquia, con cultivo negativo, así también los hemocultivos.

Se inició manejo con vancomicina, ceftriaxona, claritromicina, aciclovir y oseltamivir. Durante estancia en UCIP mediante FilmArray ${ }^{\mathrm{TM}}$ en aspirado nasal se detectó hMPV.

La Rx de tórax de control fue sugestiva de absceso o cavitación (Figura 3), y en una TAC de alta resolución de tórax se documentó cavitación apical izquierda y datos de consolidación en lóbulos inferiores (Figura 4). Se agregó piperacilina-tazobactam por sobreinfección con Pseudomonas aeruginosa (aislamiento en secreción faríngea). Posteriormente, la evolución fue a la mejoría, sin nuevos episodios convulsivos, egresando a los 33 días de hospitalización.

\section{DISCUSIÓN}

La IRA fue responsable de $20 \%$ de la mortalidad en niños menores de cinco años en el año 2000, en su mayoría en África y Asia. ${ }^{1}$ En Colombia, a la semana epidemiológica 52 de 2018, se notificaron 6,996,552 consultas externas y urgencias, 246,489 hospitalizaciones en sala general y 22,646 hospitalizaciones en UCI

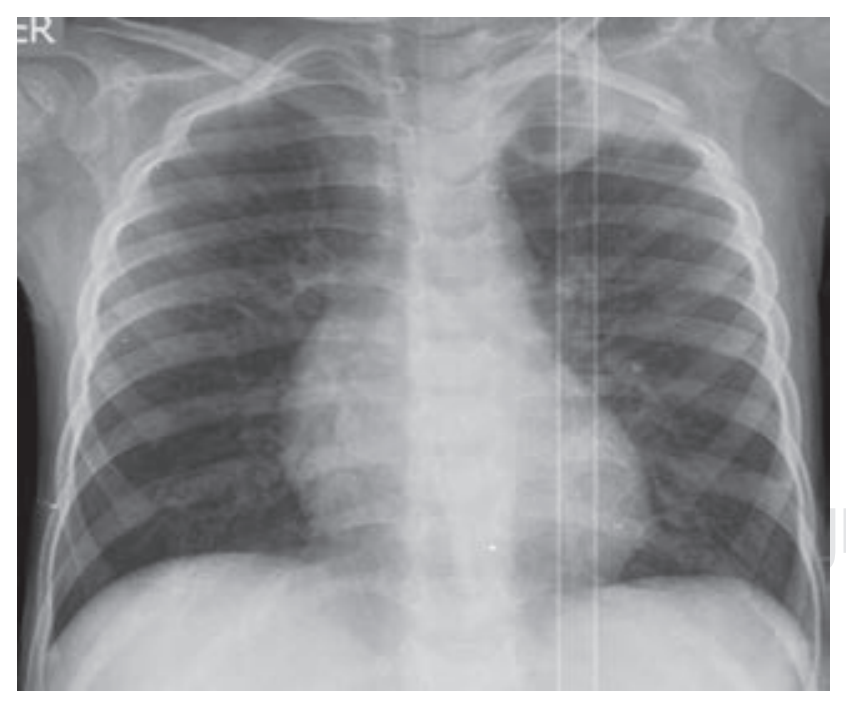

Figura 3: Rx tórax. Opacidad en el lóbulo superior izquierdo con imagen radiolúcida redondeada en su interior, compatible con consolidación y cavitación.

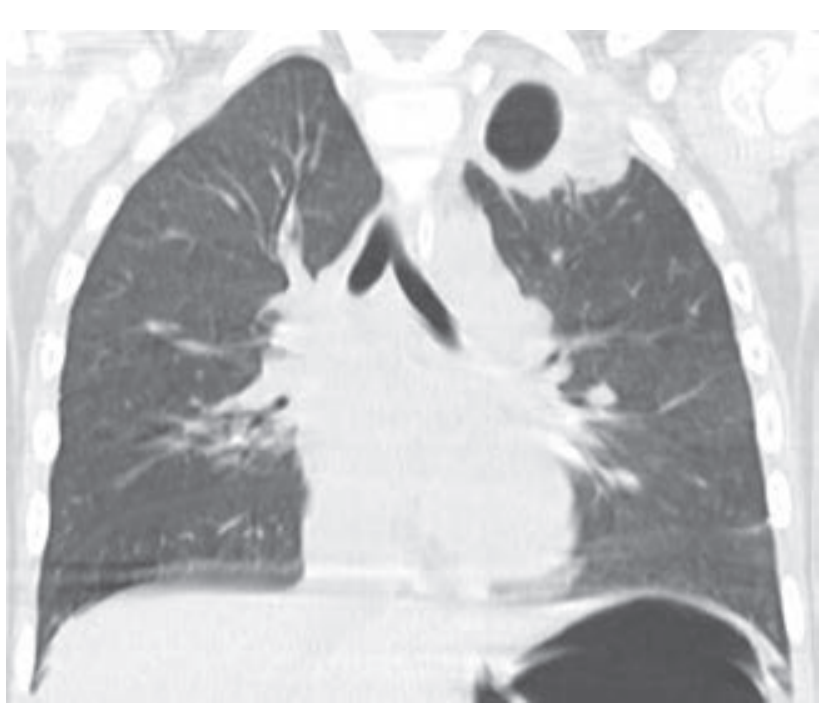

Figura 4: TACAR de tórax. Masa cavitada apical izquierda que sugiere neumonía cavitada.

por IRA. El hPMV correspondió a 3\% de 332 muestras positivas para virus respiratorios. ${ }^{6}$

El hMPV es la segunda causa más frecuente de bronquiolitis y neumonía en niños menores de cinco años ${ }^{2}$ y su prevalencia aumenta en climas con bajas temperaturas y humedad relativa, especialmente en los meses de marzo y abril. 7,8

El hMPV es un virus ARN, perteneciente al género Metapneumovirus, subfamilia pneumovirinae y familia paramyxoviridae. ${ }^{9}$ Su genotipo se divide en tipo A y B, y a su vez, estos se subdividen en A1, A2, B1 y B2 según la secuencia de glucoproteínas de superficie de unión (G) y de fusión (F): no parece existir relación entre la clasificación y la gravedad del cuadro clínico, ${ }^{1}$ motivo por el cual la identificación del mismo no se realiza de forma rutinaria. La infección por hMPV se ha relacionado a ciertas comorbilidades respiratorias, como asma y enfermedad pulmonar crónica, ya que cerca de $40 \%$ de preescolares hospitalizados con hMPV han tenido estos padecimientos. ${ }^{1,10}$

Por su parte, la infección grave por hMPV se ha asociado con prematurez, infección nosocomial, asma, cardiopatías congénitas, enfermedades neuromusculares, síndrome de Down, así como con inmunodeficiencias congénitas o adquiridas. ${ }^{1,11,12}$ Pancham y colegas realizaron un estudio con 58 pacientes con infección por hMPV, donde $32.7 \%$ nacieron con prematurez extrema, quienes tuvieron estancia hospitalaria prolongada y requirieron mayor aporte de oxígeno. ${ }^{13}$ De igual forma, Davis y su grupo reportaron que $56 \%$ de 815 pacientes 
con aislamiento hMPV tenían una enfermedad crónica de base, observando que fue más frecuente en niños de dos años, y que 18\% requirió manejo en UCIP, de los cuales $6 \%$ estuvieron bajo ventilación mecánica invasiva. ${ }^{14}$

Otro factor asociado con mayor estancia hospitalaria e ingreso a UCIP es la coinfección con otros agentes virales y bacterianos. ${ }^{15} \mathrm{El}$ virus sincitial respiratorio (VSR) es el principal, con frecuencias que van de 5\% a $17 \%$, seguido por parainfluenza, influenza, coronavirus, Streptococcus pneumoniae, Mycoplasma pneumoniae y Chlamydia pneumoniae. ${ }^{1,12,16,17}$ En los casos que presentamos no tenían alguno de estos factores de riesgo.

Las patologías causadas por hMPV corresponden principalmente a neumonía y bronquiolitis; en un estudio se detectó que el 5.5\% de 857 fue positivo para hMPV, de los cuales $36 \%$ cursó con neumonía y $10 \%$ con bronquiolitis. ${ }^{18}$ Del mismo modo, Williams y colaboradores describen 49 pacientes con hMPV, de los cuales $59 \%$ tuvo bronquiolitis, $18 \%$ croup y $8 \%$ neumonía. ${ }^{19}$ Además, se debe señalar que también se han descrito casos de otitis media aguda ${ }^{20}$ y exacerbación de cuadro de asma. ${ }^{19,21}$

Un punto a destacar es que la sintomatología que cursa con infección por hMPV es indistinguible de la producida por otros virus, como VSR, parainfluenza o influenza. ${ }^{22}$

Por otro lado, hMPV se ha asociado también con encefalitis primaria, y su sintomatología varía desde crisis febriles hasta encefalitis grave. ${ }^{4}$ Se conoce que el virus tiene la capacidad de invasión directa al SNC. ${ }^{23}$

Schildgen y su equipo reportaron un caso de encefalitis fatal en un paciente de 14 años, en quien se aisló hMPV por PCR en tejido pulmonar y cerebral, además presentó crisis convulsivas, hipertensión intracraneal, pleocitosis en el estudio del LCR y cambios en estudios de neuroimagen. ${ }^{24}$ Igualmente, Arnold y colegas describieron nueve casos de alteración neurológica temporal asociada a la presencia de hMPV aislado en vía aérea superior. Estos autores consideran que estos pacientes tienen mayor riesgo de cursar con alteraciones neurológicas que cuando hay infección por VSR. ${ }^{4}$ Los cambios evidenciados en imágenes cerebrales de pacientes con encefalitis por hMPV describen lesiones múltiples en la sustancia blanca y alteraciones corticales, tal como se reporta en el caso de un paciente con síntomas respiratorios y alteraciones neurológicas. ${ }^{5}$

No hay tratamiento específico para la infección por hMPV, por lo cual las medidas son de soporte y manejo sintomático. Se ha descrito que ribavirina en aerosol, inmunoglobulina e inhibidores de fusión y palivizumab pudieran ser empleados, sin embargo, no hay ensayos clínicos que avalen su beneficio. ${ }^{25-29}$

\section{REFERENCIAS}

1. Panda S, Mohakud NK, Pena L, Kumar S. Human metapneumovirus: review of an important respiratory pathogen. Int J Infect Dis. 2014; 25: 45-52. doi: 10.1016/j.ijid.2014.03.1394.

2. Céspedes PF, Palavecino CE, Kalergis AM, Bueno SM. Modulation of host immunity by the human metapneumovirus. Clin Microbiol Rev. 2016; 29(4): 795-818. doi: 10.1128/CMR.00081-15.

3. Freymuth F, Vabret A, Legrand L, Dina J, Gouarin S, CuvillonNimal D et al. Métapneumovirus humain. Pathol Biol. 2009; 57(2): 133-141. doi: 10.1016/j.patbio.2008.04.005.

4. Arnold JC, Singh KK, Milder E, Spector SA, Sawyer MH, Gavali $S$ et al. Human metapneumovirus associated with central nervous system infection in children. Pediatr Infect Dis J. 2009; 28(12): 1057-1060. doi: 10.1097/ INF.0b013e3181acd221.

5. Fernández IS, Polo MR, Muñoz-Almagro C, Carretero LM, Ureña SF, Muñoz AR et al. Human metapneumovirus in the cerebrospinal fluid of a patient with acute encephalitis. Arch Neurol. 2012; 69(5): 649-552. doi: 10.1001/archneurol.2011.1094.

6. Ministerio de Salud y Protección Social Colombia. Boletín epidemiológico semanal. Sistema de Vigilancia en Salud Pública. 2018. pp. 1-31.

7. Darniot $M$, Pitoiset $C$, Millière L, Aho-Glélé LS, Florentin E, Bour JB et al. Different meteorological parameters influence metapneumovirus and respiratory syncytial virus activity. J Clin Virol. 2018; 104: 77-82. doi: 10.1016/j.jcv.2018.05.002.

8. Mizuta K, Abiko C, Aoki Y, Ikeda T, Matsuzaki Y, Itagaki T et al. Seasonal patterns of respiratory syncytial virus, influenza $A$ virus, human metapneumovirus, and parainfluenza virus type 3 infections on the basis of virus isolation data between 2004 and 2011 in Yamagata, Japan. Jpn J Infect Dis. 2013; 66(2): 140-145.

9. Montejano L, Noyola D. Infecciones por metapneumovirus humano en pacientes pediátricos. Rev enfermedades. Infecc en Pediatr. 2007; 20(79): 74-79.

10. Edwards K, Zhu Y, Griffin M, Weinberg G, Hall C, Szilagyi P et al. Burden of human metapneumovirus infection in young children. N Engl J Med. 2013; 368(7): 633-643. doi: 10.1056/ NEJMoa1204630.

11. Principi N, Esposito S. Paediatric human metapneumovirus infection: Epidemiology, prevention and therapy. J Clin Virol. 2014; 59(3): 141-147. doi: 10.1016/j.jcv.2014.01.003.

12. Schildgen $\mathrm{V}$, van den Hoogen $B$, Fouchier R, Tripp RA, Alvarez $\mathrm{R}$, Manoha $\mathrm{C}$ et al. Human metapneumovirus: lessons learned over the first decade. Clin Microbiol Rev. 2011; 24(4): 734-754. doi: 10.1128/CMR.00015-11.

13. Pancham K, Sami I, Perez GF, Huseni S, Kurdi B, Rose MC et al. Human metapneumovirus infection is associated with severe respiratory disease in preschool children with history of prematurity. Pediatr Neonatol. 2016; 57(1): 27-34. doi: 10.1016/j. pedneo.2015.03.008.

14. Davis CR, Stockmann C, Pavia AT, Byington CL, Blaschke $\mathrm{AJ}$, Hersh AL et al. Incidence, morbidity, and costs of human metapneumovirus infection in hospitalized children. J Pediatric Infect Dis Soc. 2016; 5(3): 303-311. doi: 10.1093/jpids/piv027.

15. Lin P, Lin T, Huang YY, Tsao K, Huang YY. Human metapneumovirus and community-acquired pneumonia in children. Chang Gung Med J. 2005; 28(10): 683-638. 
16. Greensill J, McNamara PS, Dove W, Flanagan B, Smyth RL, Hart CA. Human metapneumovirus in severe respiratory syncytial virus bronchiolitis. Emerg Infect Dis. 2003; 9(3): 372-375.

17. Semple MG, Cowell A, Dove W, Greensill J, McNamara $P S$, Halfhide $C$ et al. Dual infection of infants by human metapneumovirus and human respiratory syncytial virus is strongly associated with severe bronchiolitis. J Infect Dis [Internet]. 2005; 191(3): 382-386. doi: 10.1086/426457.

18. Peiris JSM, Tang $\mathrm{WH}$, Chan KH, Khong PL, Guan Y, Lau $\mathrm{YL}$ et al. Children with respiratory disease associated with metapneumovirus in Hong Kong. Emerg Infect Dis. 2003; 9(6): 628-633. doi: 10.3201/eid0906.030009.

19. Williams J, Harris P, Toilefson S, Halburnt-Rush L, Pingsterhaus $\mathrm{J}$, Edwards $\mathrm{K}$ et al. Human metapneumovirus and lower respiratory tract disease in otherwise healthy infants and children. N Engl J Med. 2004; 350(5): 443-450. doi: 10.1056/ NEJMoa025472.

20. Williams J, Toilefson S, Nair S, Chonmaitree T. Association of human metapneumovirus with acute otitis media. Int $J$ Pediatr Otorhinolaryngol. 2006; 70(7): 1189-1193. doi: 10.1016/j. ijporl.2005.12.007.

21. Williams J, Crowe J, Enriquez R, Minton P, Stokes R, Hamilton R, et al. Human metapneumovirus infection plays an etiologic role in acute asthma exacerbations requiring hospitalization in adults. $J$ Infect Dis. 2005; 192(7): 1149-1153. doi: 10.1086/444392.

22. Van den Hoogen BG, van Doornum GJJ, Fockens JC, Cornelissen JJ, Beyer WEP, de Groot R et al. Prevalence and clinical symptoms of human metapneumovirus infection. J Infect Dis. 2003; 188(10): 1571-1577. doi: 10.1086/379200.
23. Niizuma T, Okumura A, Kinoshita K, Shimizu T. Acute encephalopathy associated with human metapneumovirus infection. Jpn J Infect Dis. 2014; 67: 213-215. doi: 10.7883/ yoken.67.213.

24. Schildgen O, Glatzel T, Geikowski T, Scheibner B, Matz B, Bindl $L$ et al. Human metapneumovirus RNA in encephalitis patient. Emerg Infect Dis. 2005; 11(3): 467-470. doi: 10.3201/ eid1103.040676.

25. Wyde PR, Chetty SN, Jewell AM, Boivin G, Piedra PA. Comparison of the inhibition of human metapneumovirus and respiratory syncytial virus by ribavirin and immune serum globulin in vitro. Antiviral Res. 2003; 60(1): 51-59. doi: 10.1007/s00247-017-3943-5.

26. Moe N, Krokstad S, Stenseng IH, Christensen A, Skanke LH, Risnes KR et al. Comparing human metapneumovirus and respiratory syncytial virus: Viral codetections, genotypes and risk factors for severe disease. PLoS One. 2017; 12(1): 1-19. doi: 10.1371/journal.pone.0170200.

27. Ulbrandt ND, Ji H, Patel NK, Riggs JM, Brewah YA, Ready S et al. Isolation and characterization of monoclonal antibodies which neutralize human metapneumovirus in vitro and in vivo. J Virol. 2006;80(16): 7799-7806. doi: 10.1128/JVI.00318-06.

28. Wen SC, Williams JV. New approaches for immunization and therapy against Human metapneumovirus. Clin Vaccine Immunol. 2015; 22(8): 858-866. doi: 10.1128/CVI.00230-15.

29. Schuster JE, Cox RG, Hastings AK, Boyd KL, Wadia J, Chen Z et al. A broadly neutralizing human monoclonal antibody exhibits in vivo efficacy against both human metapneumovirus and respiratory syncytial virus. J Infect Dis. 2015; 211(2): 216-225. doi: 10.1093/ infdis/jiu307. 